\title{
Plataforma ética para el mejoramiento continuo: El caso de una empresa Carbonífera en Venezuela
}

\author{
Rincón, Sorayda \\ Urdaneta, Marisela**
}

\section{Resumen}

El presente artículo tiene como objetivo estudiar los elementos teóricos sobre el concepto de ética aplicada a las empresas, asi como la relación existente entre ésta y la competitividad empresarial, con la finalidad de responder a la interrogante sobre si produce beneficios ser éticos en las empresas. Con tales propósitos se estudia una empresa carbonífera, en el Estado Zulia. Se aplicó una entrevista estructurada a cinco gerentes de la empresa analizada para determinar aspectos comunes relevantes a las variables en estudio y se analizaron los documentos estratégicos de la organización. Los resultados de la investigación muestran que la empresa ha alcanzado logros significativos en el manejo de los valores, la promoción del recurso humano, la responsabilidad social y ecológica, en la búsqueda de una plataforma ética para el mejoramiento contínuo.

Palabras clave: Ética empresarial, competitividad empresarial, dirección por valores, Empresa Carbonifera, Estado Zulia, Venezuela.

\section{The Ethical Platform for Continuous Growth: The Case of a Coal Company in Venezuela}

\author{
Abstract \\ The objective of this article is to study the theoretical elements related to the concept of ethics \\ applied in business, as well as the relationship that exists between ethics and business competition. \\ The purpose was to answer the question of whether or not being ethical is beneficial to business. For \\ Recibido: 00-04-05. Aceptado: 01-01-25 \\ * Sociólogo. Magister en Planificación y Gerencia de C y T. Profesora Agregada Escuela de So- \\ ciologia, LUZ. Profesora Asociada de Postgrado, URBE. \\ E-mail:sorarincon@hotmail.com. \\ * Lic. En Comunicación Social, Mención Impreso. Magister en Gerencia de Empresa, Mención \\ Mercadeo. Profesora Agregada de Postgrado, UABE.
}


this purpose, a coal company, operating in Zulia State (Venezuela) is studied. A structured interview was applied to five managers in the company and was analyzed to determine relevant common aspects in the variables under study. Strategic organizational documents were also analyzed. The results showed that the business had reached significant success in the management of values, the promotion of human resources, social and ecological responsibility, and in the search for an ethical platform for continuous improvement.

Key words: Business ethics, business competition, value direction, coal business, Zulia State, Venezuela.

\section{Introducción}

El interés por la ética empresarial ha crecido significativamente en paralelo con el incremento en el número de códigos de comportamiento y comités éticos en las más conocidas y exitosas empresas del mundo, hasta el punto que hay asuntos en los que una decisión se ve condicionada principalmente por los criterios éticos.

El mundo empresarial parece reconocer que la ética no es un valor añadido, sino un valor intrínseco a toda actividad económica. La clave es que las actividades empresariales concentran un cúmulo de factores humanos y los seres humanos damos a todo nuestro obrar una dimensión inevitablemente ética.

Nuestro país no debe ser la excepción y aun cuando este movimiento sólo comienza a posicionarse en la mente de nuestros empresarios, algunos asoman inicios en este proceso.

La industria minera de carbón en Venezuela, no debe estar ajena a esto, especialmente porque a fin de superar las fuertes presiones del mercado (precios bajos) y un incremento de sus costos de operación, desde el año 1996, ha iniciado un profundo proceso de transformación para lograr sobrevivir a estas circunstan- cias y poder consolidarse como una organización exitosa, en la cual todos sus participantes obtengan los beneficios de la gestión que se adelanta; es decir, que la empresa pueda convertirse en un proyecto vital.

\section{Sobre la ética empresarial: Reflexiones teóricas}

Existen distintas definiciones de la ética, elaboradas a lo largo de muchos años de estudio. Podemos diferenciar dos maneras de ver la ética: en su uso común la definiremos como un conjunto de principios u opiniones morales sobre acciones aceptables o inaceptables en un determinado campo en la actividad humana (Oceano/Centum, 1990) Es decir, como un conjunto de ideas o pensamientos sobre comportamientos aceptables o inaceptables de las personas, que determinan lo correcto o incorrecto de una acción. Esta ética se basa fundamentalmente en el "deber ser", aquello que quisiéramos todos alcanzar pero que se presenta como algo difícil que sólo puede ser obtenido por algunos grupos que están al margen de la sociedad y de las empresas, como por ejemplo: los sacerdotes, entre otros. La ética según esta conceptualización se aleja de la 
vida de las personas y se sobrepone por encima de ella como algo exterior e inalcanzable (Cortina, 1996).

En los distintos ámbitos de la vida empresarial, se ha criticado esta forma de entender la ética ya que se ha convertido en un deber ser o utopía que no puede ser llevada a la práctica; se aleja de los hombres para transformarse en una conciencia no real que los sobrepasa, que está por encima de las decisiones cotidianas (Cortina, 1996).

La base fundamental de la crítica es ¿para qué nos sirve esta ética? La respuesta pareciera ser que, vista de esta manera, no sería mas que una serie de referencias morales, preceptos o principios que raramente podrian ser aplicables a situaciones de la vida real, por lo tanto su uso estaría restringido al deber ser que quisiéramos alcanzar pero que estamos convencidos que nunca lograremos. Desde esta óptica, la ética no forma parte de la vida real y mucho menos puede formar parte de los negocios y de las empresas. Pareciera que en los negocios, si partimos del anterior concepto de ética, mas bien debemos deshacernos de los principios y valores morales como principio para ser exitosos. ¿Debemos dejar la ética en la puerta de la empresa al igual que en todos los campos de la vida cotidiana? Definitivamente la respuesta es no. Lo que debemos es entender la ética como un saber práctico que tiene que ver con la acción, como un conocimiento de vida cuyo objetivo es la convivencia, es decir, llevar la vida a su más alta expresión, con lo cual la ética pasaría de "un deber ser" a "un poder ser" que implica fuerza, impulso para realizar algo, para hacer cada día mejor la vida en conviven- cia, buscando el respeto por la vida (Cortina, 1996).

La ética forma parte de la vida cotidiana de los hombres y debe estar presente en todas y cada una de las decisiones que éste toma. No es una camisa de fuerza. Es un desafío, un reto a vivir y actuar según valores compartidos.

La ética se convierte así en una serie de conocimientos, valores, principios que serán utilizados diariamente en la toma de decisiones. No es suficiente que tengamos muy elaborados códigos de ética, lo importante es su aplicación conciente y de convicción.

En los negocios, como en cualquier otra actividad humana en donde se toman decisiones, la ética tiene una tarea fundamental.

El análisis de la toma de decisiones en el ámbito gerencial ha dado gran importancia a dos elementos fundamentales: los valores sobre los cuales se toman las decisiones y la responsabilidad social de las decisiones tomadas.

La ética empresarial, según Cortina, (1996) se define como un saber que ayuda a las personas que trabajan en la empresa a tomar decisiones prudentes y justas fundamentadas en valores morales.

En la época actual, en la cual las organizaciones juegan un papel fundamental en la sociedad, el nacimiento y consolidación de la ética empresarial permite compartir valores en la búsqueda del bien colectivo.

La ética empresarial no es una moda sino una necesidad y una realidad apremiante en las empresas. Existen razones para afirmar que la ética produce beneficios, la falta de credibilidad es un factor de riesgo en las empresas, mientras 
que la confianza se convierte en una carta que permite el aumento sustantivo de los beneficios (Mujica y Morante, 1987).

Una empresa tiene por meta satisfacer necesidades sociales sin engaños, dentro de un marco moral de respeto a los derechos de consumidores, empleados y proveedores en la búsqueda del bienestar colectivo. Esta meta alude a la responsabilidad ética de las empresas lo cual le da sentido y la legitiman socialmente.

El objetivo ético de la unidad productiva es maximizar el bienestar neto de la comunidad a la larga y en su conjunto y no la maximización del bienestar neto del empresario (Zañartu, 1987).

Actualmente, las empresas buscan transformarse en organizaciones vitales, capaces de establecer estrategias y resultados que contribuyan no sólo a su permanencia sino a su desarrollo y al proceso de alcanzar resultados que tienen que ver con su misión, visión y valores.

Las decisiones que se toman en las empresas vitales son congruentes con su proyecto vital, el cual promueven entre los miembros de la comunidad. El código de ética debe ser el eje del proyecto vital, pero debe ser llevado a la práctica y repensado permanentemente.

En estas empresas, la ética está en la base de la determinación de sus objetivos; los fines y los medios para conseguirlos pasan por un proceso de valoración y de comunicación que se da en el interior de las mismas.

Los valores y la responsabilidad son criterios para evaluar, aceptar o rechazar normas. El incumplimiento de los valores puede comportar sentimiento de culpabilidad y sanciones internas. Los valores son aprendizajes estratégicos rela- tivamente estables en el tiempo de que una forma de actuar es mejor que su opuesta para conseguir que nos salgan bien las cosas, son el eje central de la libertad humana y de la responsabilidad, constituyen elecciones deliberadas o preferencias estratégicas por unos modos de actuar frente a otros. Es decidir cual es la forma de asumir determinadas situaciones en relación a otras formas de actuar, es la elección estratégica de un determinado principio de conducta (García y Dolan, 1997).

Se conocen diferentes formas de conceptuar los tipos de valores, una de ellas especifica que existen dos tipos de valores, los que podemos llamar finales y los valores instrumentales. Entre los valores finales están los personales y los ético - sociales. Los valores instrumentales están conformados por los ético - morales y los valores de competencia. Todos ellos son de importancia capital con relación a la decisión que se quiere tomar. Son tan importantes los valores finales, personales (lo más importante en la vida para cada quien) como los ético - sociales (que se desea para el mundo), así como los valores instrumentales, ético morales (como comportarse con quienes le rodean) y los de competencia (que hay que tener para competir en la vida). No podemos afirmar que unos sean mejores que otros en términos absolutos (García y Dolan, 1997).

Así como sabemos que los valores individuales se aprenden en la infancia a partir de modelos sociales fundamentados en creencias, en las empresas la formación de valores es un fenómeno complejo que depende de múltiples variables como: las creencias y valores del funda- 
dor, las creencias y valores de la dirección actual, las creencias y valores de los empleados, la formación y la influencia de consultores, la normativa legal existente, las reglas de juego del mercado, los valores sociales de cada momento histórico y la tradición cultural de cada sociedad, entre otros (García y Dolan, 1997).

En el proceso de conformación de valores en una empresa existen los generadores de valores, los gestores de valores y los miembros del sistema de valores; sin embargo, lo importante es que todos los miembros de la empresa participen de manera democrática en la formación de los valores, de esta manera se sentirán comprometidos con la consecución de los mismos (García y Dolan, 1997).

Los miembros de una empresa tradicional acatan un conjunto de principios y valores generados unilateralmente por la dirección, solo si su cumplimiento deriva en un sistema de recompensas y castigos, pero de ninguna manera se sienten identificados con ellos y mucho menos buscan su logro de manera permanente. Por el contrario, en una empresa realmente administrada por valores, no hay mas que un jefe: los valores de la compañia, y todos y cada uno de los miembros de la empresa deben orientarse en función de esos valores los cuales deben ser puestos en práctica de manera permanente, todos los días, ese es el verdadero éxito: aplicarlos, no sólo proclamarlos.

Los procesos comunicativos que permitan alinear al personal alrededor de valores compartidos, unidos en su misión común, hacen que se logren resultados extraordinarios en la mejora de la calidad de la empresa (García y Dolan, 1997).
El campo más definitorio de la ética empresarial es el de la responsabilidad social, como una respuesta a situaciones concretas en donde están en juego valores. Determinar cuáles son las responsabilidades consecuenciales de esta toma de decisiones fundada en valores permite, si es adecuada, un crecimiento moral de quien decide y de la organización de la que forma parte. Como resultado se produce un proceso de discusión y adquisición de conceptos morales como conciencia intelectual y como acción de la misma (Llano, 1991).

La responsabilidad social de las empresas es, en los actuales momentos, vital. La solución de los grandes problemas sociales ya no depende del mundo de la política, del Estado. Ahora, la sociedad de las organizaciones, en su sentido más concreto, convierte a las empresas en un factor de moralización o de realización de la sociedad (Llano, 1991).

La ética de la responsabilidad en la cual las empresas deben tener en cuenta las consecuencias de sus decisiones, para si mismas, con los consumidores, con sus trabajadores, con los proveedores y con la sociedad en general, permitiria hacer una sociedad mas justa y equilibrada (Llano, 1991).

Las decisiones prudentes y congruentes están relacionadas con la ética de la responsabilidad. Estas decisiones se pueden denominar decisiones inteligentes.

El factor de congruencia es, en definitiva, actuar siguiendo los principios y el compromiso del proyecto vital de la empresa. La actividad empresarial tiene una responsabilidad pública ya que produce 
bienes para la sociedad y tiene además repercusiones públicas por lo que deben ser legitimadas socialmente. Lo que la legitima es satisfacer necesidades humanas con calidad (Llano, 1991).

La mayoría de los empresarios afirman que ser ético no es rentable; es decir, que no se puede ser ético sin poner en peligro las ganancias de la empresa, el cual es, en sí mismo, el objetivo primordial de la misma; sin embargo, esta idea ha venido cambiando (Etkin, 1993).

Si se puede ser ético y obtener beneficios. Es más aquellas empresas que son éticas mejoran su productividad y permanencia en el mercado hasta el punto de ser más competitivos. En una situación de absoluta incertidumbre las únicas empresas que sobreviven y que incluso prosperan son las éticas y casi por definición, las empresas que no se comportan éticamente son precisamente las que desaparecen (Cortina y Conell, 1998).

Hoy, la competitividad tiende a ser entendida como la capacidad de una empresa de generar beneficios suficientes para mantenerse en el mercado a largo plazo, permitiéndole ser perdurable. Para ello, su base de acción debe ser un comportamiento ético. Las empresas competitivas son éticas porque merecen credibilidad y generan confianza, dos elementos a tomar en cuenta en el actual ambiente de incertidumbre empresarial. Ambos generan ventajas competitivas, fomentan la innovación, la imaginación y la apertura de nuevos mercados (Seanone, 1998).

Vemos asi que los principios morales lejos de constituirse en una barrera para los negocios se orientan a conseguir las mejores actuaciones posibles. La ética en las empresas no es una moda, sino una necesidad ante situaciones que la empresa enfrenta dia a día, no se limita a la provisión de un complejo cúmulo de consecuencias, ni a una operación de cálculo. La decisión de asumir la ética en las decisiones empresariales resulta de darle importancia a los principios éticos y las consecuencias de los actos evita el abandono de las responsabilidades sociales de la misma (Cortina, 1996).

\section{Caracterización ética en una empresa Carbonífera}

A continuación se realizará un análisis de los principales valores que rigen esta organización carbonífera ubicada en el estado Zulia, tomando en cuenta los principios y valores que la rigen y comparándolos con algunos indicadores de gestión que maneja.

\subsection{Decisiones: una labor compartida}

Se ha planteado constantemente que la cuestión de la ética está en base de los objetivos de una decisión. También es conocido que el control de cualquier actividad o trabajo implica el control del proceso. Ortiz (1998), expresa en su texto La Hora de la Ética Empresarial que antes de tomar una decisión, una actitud realista buscará tener lo más claro posible tanto el punto de partida como lo que se puede llegar a conseguir. $Y$ eso requiere hacerse cargo. Por una parte, saber qué hay que recordar, y qué conviene olvidar; de qué hay que tener buena memoria y de qué mala memoria; qué cosas arregla el paso del tiempo, y cuáles no escuchar. Escuchar no es algo pasivo: implica una 
actividad. Estar convencido de que los demás tienen cosas interesantes que aportar a un grupo, hacerse cargo, dejarse aconsejar. Descubrir cuál es el lugar más idóneo para cada persona. $Y$ hacerse entender. Saber adaptar los objetivos a la realidad existente, de modo que resulten asequibles y motivantes.

En el proceso de toma de decisiones en la empresa analizada, el tomar la decisión de los factores que impactan la organización, está concentrado para el alto nivel gerencial / directivo de la organización. Un caso que ilustra este aspecto es el proceso de preparación del plan de Negocios de la empresa. (Plan Quinquenal) En éste, la Junta Directiva establece los lineamientos estratégicos, los cuales son informados al equipo gerencial para que elabore los programas gerenciales que soporten el alcance de los objetivos corporativos. Es decir, que el equipo gerencial establece la relación entre los fines y los medios para alcanzar esos objetivos, sobre la base de la escala de valores que cada uno de ellos maneja en el marco referencial de los valores establecidos en la organización y de las metas propuestas. Una oportunidad de mejora en este proceso sería, por ejemplo, que el establecimiento de los objetivos corporativos de la empresa fuera logrado en una doble vía de valoración y comunicación donde la participación de los actores principales en el liderazgo medio del negocio tuvieran un mayor nivel de involucramiento. Este ejercicio generaría mayor compromiso en el alcance de los mismos. Es bueno recordar que la ética es un saber de alternativas y la alternativa es parte de la práctica cotidiana de la toma de decisiones gerenciales, las cuales en la práctica serán las que permitan alcanzar los objetivos corporativos de la institución.

Otro factor importante a evaluar en este importante proceso es la comunicación. Los planes de una organización establecen la orientación, acciones y programas para alcanzar las metas de la organización y una de las claves del éxito de un buen plan es el conocimiento que sobre él deben tener todos los que intervienen en el logro de sus metas. La decisión ética para garantizarlo es la implantación de un proceso de comunicación, lo cual, a la fecha, no se realiza en todos los niveles. Si bien es cierto que el equipo gerencial es informado sobre el Plan definitivamente aprobado por los accionistas de la empre$\mathrm{sa}$, no se ejecutan programas comunicacionales que transfieran esta información a todas las nóminas. Como resultado el plan pudiera no generar el compromiso, la identificación y la motivación necesarias para alcanzar sus objetivos.

\subsection{El recurso humano: tarea común de la organización}

En el propio trabajo uno desea ser considerado ante todo como persona. $Y$ la empresa, cuando es entendida como una tarea común entre hombres, manifiesta la preeminencia del capital humano sobre cualquier otro factor de producción (Ortiz, 1998).

La empresa analizada, cuenta con una fuerza - hombre de mas de 1.000 personas distribuidas en las nóminas. Aproximadamente el $75 \%$ es nómina dia- 
ria proveniente de la zona en la cual la empresa desarrolla sus actividades (Municipio Mara).

Desde el punto de vista de la compensación salarial, la empresa administra dos sistemas: nómina mensual y nómina diaria. La primera es orientada, según políticas y normas de la organización, por un Sistema de Meritocracia, administrado por el Departamento de Recursos Humanos y aprobado por el Comité Ejecutivo siguiendo los parámetros presupuestarios en el ejercicio de Presupuesto Anual aprobado por la Junta Directiva. La nómina diaria se rige por la Convención Colectiva de Trabajo cuya duración es de dos años. Aparte para elaborar una caracterización ética en el sentido expuesto, son muchos los valores que rodean una adecuada distribución de los beneficios: claridad, equidad y justicia, por ejemplo. En este caso la empresa ha decidido ubicarse en una posición de la banda salarial de sus homólogas en mercado laboral del país, con lo cual se indica una intención ética hacia su recurso humano, de mantener equidad y justicia hacia sus empleados. A pesar de la situación país y la del mercado donde la empresa desarrolla sus actividades, lo cual la ha llevado a una difícil situación económica -como muchas otras organizaciones venezolanas- la empresa ha mantenido sus criterios básicos de meritocracia hacia su personal. Quizás los porcentajes por incrementos puedan haber disminuido, pero siempre han mantenido los principios de meritocracia que orientan el sistema. Es importante destacar que-en paralelo- los accionistas -como parte de su cuota de sacrificio no han distribuido ganancias de años anteriores con lo cual están cubrien- do el déficit que el desempeño de la empresa pudiera haber generado durante esta época. Asimismo, ha mantenido el paquete de beneficios socio-económicos de sus trabajadores.

Otro factor de evaluación frecuente en la vida ética de las empresas en lo que su recurso humano se refiere, es el sistema de evaluaciones de desempeño y promociones. Ortiz (1998) explica que sea cual sea el sistema -libre designación, concurso de méritos, por evaluación interna o externa - es muy importante que el procedimiento sea conocido por los empleados. La claridad y flexibilidad son aspectos éticos básicos en la selección y promoción del personal. Y como es obvio, están muy relacionados con la necesidad de una comunicación interna suficientemente fluida y accesible.

La empresa, basa su Sistema de Evaluaciones de Desempeño en un Sistema de Meritocracia adaptado del utilizado por uno de sus accionistas. Los resultados de este sistema, son conocidos en sus principios generales, por los trabajadores de la organización, a través de la entrevista de actuación que cada supervisor debe tener al final del ciclo con su supervisado. Es fundamentalmente conocido el instrumento que recoge la información sobre la actuación y desarrollo de carrera de cada trabajador, y las acciones salariales y de promoción a corto plazo.

El número y complejidad de los asuntos que deben abordar las áreas de recursos humanos de las empresas se incrementa progresivamente. Esto se debe, básicamente, a que el personal directivo esta cada vez más conciente de lo complicado que resulta gestionar el ma- 
yor de los activos que poseen sus empresas: el capital humano.

Otro asunto importante de abordar en torno a este importante recurso de la organización, el humano, es la cuestión de la seguridad. En no pocas ocasiones los manuales al uso en ética empresarial ofrecen una disyuntiva irreconciliable: 0 se actúa de acuerdo con las propias convicciones sin mirar a las circunstancias transitorias, o sólo se tiene en cuenta el cálculo de las consecuencias que van a generarse olvidándose de los valores que privadamente puedan profesarse. Esta disyuntiva tiene desde luego una larga historia, y aflora con frecuencia en los momentos críticos en los que hay que dar una respuesta (Ortiz, 1998).

El issue Seguridad en la empresa es un tema de especial atención. Encabeza los objetivos corporativos del negocio: Cero accidentes como meta, ubicado como primer objetivo de sus planes a todo nivel. Organizacionalmente, es gerenciada desde la Coordinación Corporativa de Ambiente, Higiene y Seguridad la cual depende del Vicepresidente de Operaciones de la empresa. En cada una de las áreas operativas funciona un departamento de Seguridad. Como parte del Programa de Transformación de la empresa, los planes en materia de seguridad se han intensificado, inclusive, en la actualidad, se está tratando de implantar un sistema de valores basados en principios básicos de seguridad como: liderazgo de la alta gerencia y de todos los niveles supervisorios, actualización de los equipos de seguridad personal, aceptación de responsabilidad personal por aporte de cada uno de los trabajadores, orden y limpieza como base para todas nuestras ac- tividades, entrenamiento intensivo en materia de prevención de accidentes y consolidación y optimización de un sistema para reportar accidentes.

Con la implantación de este sistema (a partir de Mayo de 1999) el índice de ocurrencia de accidentes de equipos se ha reducido en un $40 \%$ y se han logrado importantes records de seguridad en diferentes áreas de la organización. Los planes de la empresa muestran conciencia de las oportunidades de mejora detectadas en esta área conectadas a acciones definidas para solventar las debilidades que todavía puedan tener en este sentido. La organización demuestra una preocupación ética el fundamentar la política de seguridad en la implantación de unos principios y valores que orientan los programas de seguridad de la empresa. Este esfuerzo es reconocido por todas sus nóminas, especialmente, la de los operadores de los equipos de minería quienes afirman haber recibido el impacto positivo de las acciones tomadas por la empresa en torno a algo tan crítico como la vida de sus trabajadores.

\subsection{Del compromiso legal a la conciencia ecológica}

Para conseguir que el desarrollo de una organización sea sustentable por las actuales generaciones y las futuras, se precisa, fundamentalmente un cambio profundo de mentalidad. Los recursos naturales no pueden ser explotados indiscriminadamente, no sólo porque son limitados, sino porque una actitud de ese tipo dejar ver claramente que se está olvidando que el ser humano es parte integrante, vital, de este sistema y que la ecologia y 
el medio ambiente son un derecho de los seres humanos. Las empresas van incorporando a sus estrategias la necesidad de compatibilizar su desarrollo con el respeto a la naturaleza. $Y$ cada vez son mayores las exigencias de estudio previo que demuestran que ya ha pasado el tiempo en que la preocupación ecológica pudo haber sido simplemente un valor añadido (Ortiz, 1998).

La industria minera es, por razones técnicas, impactadora del medio ambiente donde se lleva a cabo. En el caso del carbón, el proceso de extracción se inicia con la remoción de la capa vegetal y del material estéril ubicado en la parte superior de los mantos. Este asunto, para cualquier ecologista resulta en una ecuación directa al resultado de empresa no ética por su alto impacto en la afectación del medio ambiente. Sin embargo, ¿no sería una decisión no ética dejar este recurso en la tierra partiendo de la base del posible desarrollo socio - económico que puede dejar en la zona donde se realiza la actividad? Obviamente, es una cuestión de evaluar ambos aspectos y colocarlos en una balanza para determinarlo.

Se consultó a los líderes de los procesos orientados a la preservación del ambiente dentro de la organización quienes explicaron algunos puntos interesantes: La empresa, ha fundamentado su filosofía gerencial sobre una tríada de desarrollo sustentable; el desarrollo económico, el desarrollo social y el desarrollo ambiental. Con relación a este último, afirman que han intensificado las acciones orientadas a optimizar el desempeño ambiental, minimizar posibles impactos y consolidarse como una empresa mundialmente reconocida en esta área. To- man un marco legal que así lo impone y un compromiso ético social que lo determina. Según publicaciones periódicas corporativas, la organización invirtió -solo en la mina más de $\mathbf{4 5 0}$ millones de bolivares en programas ambientales para 1998. Aun cuando tienen más de 2.100 hectáreas permisadas, han intervenido solo unas 1300 hectáreas. Tienen un programa de recuperación de áreas explotadas que ya tiene el modesto resultado de unas 190 hectáreas reforestadas con especies de la zona y con otras que han traído de otras áreas con la idea de mejorar el proceso, sin que esto signifique modificaciones fuertes a las especies del lugar. Cumplen con toda la normativa legal en este sentido y son supervisados, muy de cerca por los reguladores en esta materia: el Ministerio del Ambiente y los Recursos Naturales Renovables, el Ministerio de Energía y Minas y el ICLAM. Este equipo manifiesta estar conscientes de la labor que tienen por delante en la recuperación de las áreas afectadas y continuarán trabajando en este sentido.

Es evidente que la organización realiza un esfuerzo en esta materia y muestra en documentos, declaraciones y algunas acciones muestras de un compromiso ético con este factor. Está además condicionada legalmente por los controles cada vez más rigurosos que el Estado y la Sociedad Civil impone en este sentido.

El medio ambiente es un factor plenamente integrado en las políticas, y se incluyen en todos los códigos éticos, tal como lo expresa Ortiz(1998) y como tal debe ser parte de la convicción de una organización cuya actividad es tan impactante como la explotación de los minera- 
les, pasando de ser un mero cumplimiento legal a una muestra de la sensibilidad ecológica que es base para la preservación de un mundo habitable para las generaciones del futuro.

\subsection{Bajo el principio de responsabilidad social}

En nuestros días, más que nunca, las grandes compañias gozan de la posibilidad de regenerar el tejido social. $Y$ el argumento ético está claro: si pueden hacerlo, deben hacerlo (Ortiz, 1998).

En todas las épocas se han dado modelos de organización de empresarios deseosos de contribuir a una justa distribución de la riqueza. Ejemplo de ello es la preocupación actual de algunas empresas por devolver a la sociedad una parte de sus ganancias mediante la promoción de beneficios sociales para sus empleados o a través de actividades de inversión social o, mejor conocidas, como programas de donaciones.

Pero esas responsabilidades sociales, si bien loables e importantes, no son la base real de un concepto ético de responsabilidad social. Porque junto a esas posibles contribuciones voluntarias, la empresa encierra en su mismo funcionamiento un conjunto de objetivos sociales irrenunciables: el aumento del empleo, la mejora de las condiciones laborales -salario, formación- protección del medio ambiente, desaparición de tratamientos discriminatorios, redistribución de la riqueza. Lo accidental no puede enmascarar lo esencial (Ortiz, 1998).

La empresa, expresa en su documento de filosofía gerencial, con relación al desarrollo sustentable y la res- ponsabilidad social, que se afianzará el compromiso ético con las poblaciones bajo influencia operacional, a través del apoyo que la empresa brinda a las iniciativas comunitarias que promueven el desarrollo de la zona. Por otra parte, en su presentación de resultados se muestran como logros de responsabilidad social: generación de unos 1.100 empleos directos y más de 1.500 indirectos, construcción de viviendas en su zona de influencia, pago por concepto de royalty por mas de 13 mil millones de bolivares para el año 2000, pago de impuestos municipales por las de 1.300 millones de bolivares y programas de inversión social por más de 150 millones de bolivares con un monto similar recursos apalancados a través de programas de co-gestión con otras instituciones.

\subsection{Comunicación de los valores}

La información emitida va acompañada de un conjunto de aspectos difícilmente explicables, pero que sin duda matizan los contenidos objetivos. Sobre qué debe ser informado cada componente de una organización? puede decirse que, es lo que sea justo, es aquello a lo que tenga derecho y definitivamente los valores de la empresa es información no catalogada justamente como confidencial. De hecho, para generar los efectos de identificación, motivación, actitudes y conductas productivas, se requiere como principio fundamental un proceso de comunicación exhaustivo sobre su contenido e implicaciones.

La organización en estudio cuenta con un sistema de valores implantado en 1993, el cual establece nueve valores 
como principios rectores de su conducta organizacional.

En la evaluación ética de esta área se pudo determinar:

- Se incluye a la ética como un valor de la organización. En todo caso, ética es el marco referencial dentro del cual se inscriben los valores establecidos por una organización.

- Aun cuando el proceso de elaboración de los valores no incorporó la participación de todos los miembros de la organización, que es la tendencia moderna de la gestión gerencial, los valores -preparados en el departamento de Recursos Humanos, presentados y aprobados por el Comité Ejecutivo de la empresa-fueron distribuidos, acompañados de una comunicación firmada por la presidencia, a todos los trabajadores de la empresa. Su proceso de información fue reforzado por la inclusión de un valor en cada edición de la publicación mensual de la institución. Este proceso de internalización de los principios se mantiene a través de los diferentes instrumentos comunicaciones de la empresa.

- Sin embargo, un significativo porcentaje de los empleados de la empresa no conoce los valores de la organización y otros aunque, reconocen la existencia de "un libro de principios y valores" no puedan recordar mas de dos de los enunciados. Surge acá una importante oportunidad para revisar y mejorar este proceso.

- En el entorno local de la empresa (proveedores / comunidad) no se conocen los valores que rigen a la organización, ni siquiera aquellos vincula- dos con las relaciones comerciales y la responsabilidad social.

\subsection{Relación valores - desempeño de la organización}

La aceptación de un código ético, por tanto, es algo más que una declaración de buenas intenciones ya que nos introduce en una perspectiva moral diferente; representa unos contenidos objetivos fijos, admitidos por todos, no negociables, gracias a lo cual una actuación éticamente correcta pasa de entrañar un cierto riesgo - el riesgo de quedarse sóloa ser socialmente reconocida y premiada (Ortiz, 1998).

El código de ética de la empresa analizada, muestra importantes principios y compromisos establecidos con todos los participantes del negocio. Sus indicadores de desempeño en algunos casos lo confirman.

Por ejemplo, el indicador costo de labor representa un porcentaje significativo de su costo unitario con lo cual pudiera inferirse que la empresa invierte considerables sumas de dinero en compensación $y$ beneficio de sus empleados, soportando, desde el punto de vista económico y cuantitativo, valores expresados en su código tales como responsabilidad y profesionalismo.

El área responsabilidad social, en la cual se incluye ambiente, seguridad e inversión social, suma un costo de $0.6 \%$ del costo unitario total. Sin embargo, adicionalmente deben incorporarse el royalty $(10 \%$ del valor FOB) y el impuesto municipal ( $1.4 \%$ del valor FOB) que si bien pudieran clasificarse como obligaciones 
de la empresa, suponen un impacto directo en los factores de desarrollo social en la zona, es dinero que se queda en el estado Zulia. Valores como respeto por todos los participantes del negocio (proveedores, clientes, trabajadores, comunidad y accionistas entre otros) están vinculados con este indicador.

Por su parte, los indicadores relativos a accidentes e incidentes en seguridad y ambiente, traducen que en esta área, se realizan sólidos esfuerzos por mejorar y pueden reforzarse las acciones proactivas orientadas a la prevención de la ocurrencia de los mismos, sobre todo por los valores tan altos que involucran respecto a la vida presente y futura de trabajadores y habitantes de la zona. El profesionalismo, respeto, responsabilidad y sentido de pertenencia son valores que orientan este indicador de desempeño en la organización.

\section{Conclusiones}

Luego de terminada nuestra investigación, podemos concluir que la ética no es una moda; es ante todo una necesidad muy apremiante en las organizaciones del mundo actual, globalizado y altamente competido.

Cada día más inversionistas se están convenciendo de la necesidad de invertir en negocios que además de mostrar solidez económica a través de sus activos, flujos de caja, estado de ganancias y pérdidas e indicadores financieros, cuenten con una conciencia ambiental y social y un enfoque positivo hacia principios y valores que beneficien el entorno en el cual se desarrollan.
La empresa analizada no puede en la actualidad ser calificada como una empresa que cumple $100 \%$ los atributos y condiciones de una organización ética de su proyecto vital. Se encuentra en un nivel de supervivencia, haciendo fuertes esfuerzos por sobrevivir en un mercado de precios bajos, a través de un proceso de reducción de sus costos operativos, pero manteniendo sus programas ambientales, laborales, de seguridad y responsabilidad social.

La empresa ha iniciado algunas acciones para construir una plataforma ética para su proceso de mejoramiento, pero su camino es todavía largo y debe focalizarse en actividades gerenciales orientadas por la verdadera implantación de un sistema de valores que propicie la motivación, identificación y responsabilidad de generar productividad y rentabilidad. Debe tomar la oportunidad de la situación plantea. da y formular su proyecto vital.

\section{Propuesta: Hacia una plataforma ética para el mejoramiento continuo}

En esta sección trataremos de introducir algunos elementos claves que nos permitirán perfilar las bases de la propuesta:

a. Reforzar la comunicación e información sobre conceptos éticos como la honradez, lealtad, solidaridad, fidelidad y transparencia en las relaciones con sus proveedores y entorno en general.

b. La empresa debe consolidar su reconocimiento al respeto, la dignidad y las necesidades del otro. Esto le per- 
mitirá mejorar sus relaciones con las personas como fines en sí mismos y no como medios.

c. La empresa puede profundizar su conciencia de responsabilidad social, no únicamente referido al impacto comunitario, ecológico o ambiental, sino a otros asuntos en los cuales también puede ser líder, responsable y agente de la socialización. Además de la inversión de recursos económicos, su gestión de cooperadora del desarrollo le validará en el tiempo como una organización de profundas convicciones éticas en el mundo empresarial.

d. Profundizar en el proceso de convicciones en los trabajadores, ya que las personas convencidas desde dentro se coordinan porque están identificadas con un proyecto vital.

e. Establecer u optimar las relaciones éticas con todos los grupos de interés dentro y fuera de la empresa. Estas relaciones deben continuar caracterizadas por la corresponsabilidad, comunicación y transparencia.

f. Reforzar la política de comunicación interna a través de las cuales se discutan los principios y valores que constituyen el código ético y donde participen todos los que conformen la empresa con la finalidad de que todos se sientan responsables de cumplirlos.

Para institucionalizar esta propuesta dentro de la empresa se sugiere la creación de un comité ético conformado por directivos, personal gerencial, empleados y grupos de interés del entorno de la organi- zación para formular cuáles deben ser las orientaciones que encaminen la empresa el mejoramiento permanente basado en una dirección por valores.

\section{Bibliografía citada}

Cortina, Adela (1996). Ética de la Empresa. Editorial Trotta, Madrid.

Cortina Adela y Conell, Jesús (1998). Democracia Participativa y Sociedad Civil. Una Ética Empresarial. Fundación Social Siglo del Hombre, Bogotá.

Etkin, Jorge (1993). La Doble Moral de las Organizaciones. Editorial Mc Graw Hill, Madrid.

García, S y Dolan, S. (1997). La Dirección por Valores. Editorial Mc Graw Hill, Madrid.

Llano, Cartos (1991). El Empresario ante la Responsabilidad y la Motivación. Editorial Mc Graw Hill, Madrid.

Mujica, Patricio y Morante, Fellpe (1987). ¿Conflictos o Amonía?, en Ética Economía compilado por Richards H. Editorial Tecnológica, San José de Costa Rica.

OCÉANO/CENTRUM (1990). Enciclopedia del Management Ético en la Dirección de Empresas. Edición Española, Barcelona, España.

Ortiz, José Maria (1998). La Hora de la Ética Empresarial. Serie Mc Graw Hill de Management, Colombia.

Seanone, Juan Carlos (1998). Ética y Economia. Editorial El Borum, Buenos Aires, Argentina.

Zañartu, Mario (1987). El Ethos Requerido para una Transformación Productiva con Equidad, en Ética Economía compilado por Richards $\mathrm{H}$. Editorial Tecnológica, San José de Costa Pica. 